\title{
Matching of images with projective distortion using transform invariant
}

\section{low-rank textures}

\author{
Qiang Zhang ${ }^{\mathrm{a}, \mathrm{b} *}$, Yajun $\mathrm{Li}^{\mathrm{b}}$, Rick S. Blum ${ }^{\mathrm{c}}$, Peng Xiang ${ }^{\mathrm{b}}$ \\ ${ }^{a}$ Key Laboratory of Electronic Equipment Structure Design (Xidian University), Ministry of Education, Xi'an Shaanxi 710071, China \\ ${ }^{b}$ Center for Complex Systems, School of Mechano-electronic Engineering, Xidian University, Xi'an Shaanxi 710071,China \\ ${ }^{c}$ Electrical and Computer Engineering Department, Lehigh University, Bethlehem, PA 18015, United States
}

\begin{abstract}
A matching method is presented for images with projective distortion based on transform invariant low-rank textures (TILT). In the method, the problem of matching images with projective distortion is first reduced to a problem of matching two rectified images just with scaling and translation distortions via TILT. Then a point-feature based matching method is employed to establish the corresponding points between the two rectified images. This is different from some traditional methods that try to directly seek local affine or projective invariants from input images. Moreover, no prior knowledge on the epipolar geometry is required in the proposed method. An automatic low-rank texture region detection method is presented to make the method more applicable in practice. Additionally, a new descriptor is constructed by combining a proposed geometric shape descriptor and the traditional SIFT descriptor to further improve the correct matching rate. Experimental results demonstrate the validity of the proposed method.
\end{abstract}

Key words: Image matching, Projective distortion, Transform invariant low-rank textures (TILT), Automatic low-rank texture region detection, Geometric shape descriptor.

\section{Introduction}

Image matching is an approach to identify the corresponding points in two images of the same scene taken at different times, from different sensors or from different viewpoints [1]. It has been widely applied in many computer vision and pattern recognition tasks, including object recognition [2][3], image stitching [4], broadcast video analysis [5][6], and 3D reconstruction [7-9]. Image matching methods have been previously developed [10-16]. They are very effective at finding matches in images with a limited number of distortions, such as similarity or affinity. However, real images often include more general distortions. In fact, real images with distortion that is well approximated by projective distortion are common.

If we restrict ourselves to planar scenes, the projective distortion between two images could be described by a plane projective transformation [17][18], i.e.,

$$
\mathbf{p}^{\prime}=\left[\begin{array}{c}
x^{\prime} \\
y^{\prime} \\
w^{\prime}
\end{array}\right]=\left[\begin{array}{lll}
h_{11} & h_{12} & h_{13} \\
h_{21} & h_{22} & h_{23} \\
h_{31} & h_{32} & h_{33}
\end{array}\right]\left[\begin{array}{l}
x \\
y \\
1
\end{array}\right]=H \mathbf{p},
$$

\footnotetext{
* Corresponding author. Address: P. O. Box 183, Department of Automatic Control, Xidian University, No.2 South TaiBai Road, Xi'an, Shaanxi Province, 710071, China. Email address : qzhang@xidian.edu.cn (Q. Zhang).
} 
where the homography matrix $H$ is a $3 \times 3$ non-singular matrix. In $(1), \mathbf{p}=[x, y, 1]^{T}$ denotes the homogenous

coordinates of a point in the first image with coordinates $(x, y)^{T}$, while $\mathbf{p}^{\prime}=\left[x^{\prime}, y^{\prime}, w^{\prime}\right]^{T}$ denotes the homogenous coordinates of the corresponding point in the second image with coordinates

$$
\left[\begin{array}{c}
x^{\prime} \\
w^{\prime} \\
y^{\prime} \\
w^{\prime}
\end{array}\right]=\left[\begin{array}{l}
\frac{h_{11} x+h_{12} y+h_{13}}{h_{31} x+h_{32} y+h_{33}} \\
\frac{h_{21} x+h_{22} y+h_{23}}{h_{31} x+h_{32} y+h_{33}}
\end{array}\right] .
$$

As discussed in [17], the projective transformation is a general non-singular linear transformation of homogeneous coordinates and combines the affine transformation with projectivity. Accordingly, the problem of matching images with projective distortion is more challenging than that of matching images with similarity or affine transformation.

Some approaches to the matching of images with projective distortions have been published in recent works, among which the local-invariant based methods are one of the most popular categories. Furthermore, one of such methods is based on the assumption that the projective transformation can be locally approximated by a similarity or affine transformation. In these cases, some scale or affine invariants could be applied, such as the Scale Invariant Feature Transform (SIFT) [19], the Affine-SIFT (ASIFT) [20], and the method of Maximally Stable Extremal Regions (MSER) [21]. However, in some cases the projective transformation cannot be approximated by a similarity or affine transformation. Some projective invariants have also been proposed to overcome this problem, which are defined on point sets [18] [22], on sets composed both from points and straight lines [23] [24] or on a shape basis [25].

In addition to those using local invariants for image matching, some methods were presented which employed geometric constraints among correspondences [26-29]. These methods first defined a proper measure of the geometric consistency among point correspondences, and then formulated the image matching problem as an optimization problem [23]. Usually, these methods achieve higher performance, but also have higher computational complexity. Moreover, an accurate estimation of the epipolar geometry is usually required in these methods [26][27], either through prior knowledge of the configuration or through a few strong matches to estimate the fundamental matrix.

In the past few years, sparse representation and low-rank representation [30-32] have attracted significant attention in the field of computer vision and image processing. More especially, Zhang et al. [33] proposed a new tool, transform invariant low-rank textures (TILT), based on sparse and low-rank matrix decomposition, to recover the "intrinsic" low-rank textures in the 3D scene from the deformed 2D images. Furthermore, it can remove the deformation caused by affine or projective transformation to produce a clean image captured from a front-viewpoint. Their experiments demonstrated that TILT could effectively and robustly work for a wide range of regular and near-regular patterns or objects in real images. So far, it has been successfully applied to many computer vision tasks ,such as text extraction [34], camera calibration [35] and image rectification [36]. 
In this paper, we will present a method to address the matching of "low-rank texture images" with projective distortion by using TILT. Similar to those in [26] and [27], we first rectify the reference image and the image to be matched, respectively, to reduce the projective distortion between the two original input images to some extent. Then we exploit a point-feature based matching method on the two rectified images to obtain the final corresponding points. We achieve the rectification of the input images via TILT, instead of employing any prior knowledge on the epipolar geometry as in [26] and [27]. Experimental results demonstrate that the proposed method performs better than some existing methods such as SIFT, ASIFT and MSRE, especially in cases with images experiencing severe projective distortions.

The main contributions of this paper are as follows: (1) A matching method for images with projective distortion is proposed based on TILT. Different from those traditional methods that try to directly seek local affine or projective invariant features from the input images, the proposed method reduces the problem of matching images with projective distortion to a problem of matching images with translation and scale distortions via TILT. Moreover, it requires no prior knowledge on the epipolar geometry as in [26] and [27]. (2) An automatic low-rank texture region detection method is presented. With the proposed texture region detection method, the low-rank textures will be automatically, rather than manually as in [33], selected from the original input images before they are fed into TILT. (3) A novel descriptor is introduced for each considered point when the two rectified images are matched. In addition to the local information from each considered point, the geometric shape information of the pixels around each considered point is employed in the proposed descriptor.

The rest of the paper is organized as follows. Section 2 gives a brief introduction to TILT. In Section 3, the proposed image matching method is described in detail. Experimental results and some conclusions are given in Section 4 and Section 5, respectively.

\section{Transform invariant low-rank texture (TILT)}

To improve the readability of this paper, the key idea of TILT, presented in [33], will be reviewed briefly in this section.

Considering a 2D texture image $I_{0}$, represented by a matrix (also denoted by $I_{0}$ for convenience) of order $m_{1} \times m_{2}$, it is seen as a low-rank texture if the rank of the matrix $I_{0}$ is far less than its smaller dimension $\left(\begin{array}{lll}m_{1} & \text { or } & m_{2}\end{array}\right)$ [33]. As discussed in [33], although the planar surfaces or structures in the 3D scene exhibit low-rank textures, their images do not necessarily have low rank because of deforming transformations and corruptions.

Given a deformed and corrupted image $I$ of a low-rank texture

$$
I=\left(I_{0}+E\right) \circ H^{-1}
$$

\footnotetext{
${ }^{1}$ As discussed in [33], the low-rank texture images generally denote those containing large a number of regular or symmetrical structures.
} 
the goal of TILT is to recover the "intrinsic" low-rank texture $I_{0}$ and the transformation $H: R^{2} \rightarrow R^{2}$. In (3), $E$

denotes the corruptions and is assumed to be sparse [33]. And $I \circ H$ denotes the transformed version of the image $I$ using the transformation matrix $H^{2}$. Mathematically, the problem of TILT is formulated as

$$
\min _{I_{0}, E, H} \operatorname{rank}\left(I_{0}\right)+\gamma\|E\|_{0} \quad \text { s.t. } I \circ H=I_{0}+E
$$

where $\|E\|_{0}$ denotes the $l_{0}$-norm of the matrix $E$ (i.e., the number of the non-zero entries in the matrix $E$ ), and $\gamma>0$ is a weighting parameter that trades off the importance of the rank of the texture and the sparsity of the error.

The non-convex rank and $l_{0}$-norm minimization problems in (4) can be, respectively, relaxed by the convex nuclear-norm and $l_{1}$-norm minimization [33][37]. The nonlinear constraint function $I \circ H=I_{0}+E$ can also be linearized as $I \circ H+\nabla I \Delta H=I_{0}+E$, where $\nabla I$ is the Jacobian of the image $I$ with respect to the transformation parameters $^{3}$. Thus the problem in (4) reduces to the following convex optimization problem

$$
\min _{I_{0}, E, H}\left\|I_{0}\right\|_{*}+\gamma\|E\|_{1} \quad \text { s.t. } I \circ H+\nabla I \Delta H=I_{0}+E
$$

where $\|\cdot\|_{*}$ denotes the nuclear-norm of a matrix and is defined as the sum of its singular values. $\|\cdot\|_{1}$ denotes the $l_{1}$ -norm of a matrix and is defined as the sum of the absolute values of its entries. The optimization problem in (5) can be effectively solved by using an iterative linearization scheme. More details are provided in [33].

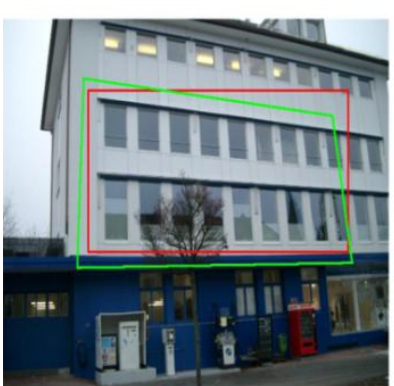

(a) Input(rank=91)

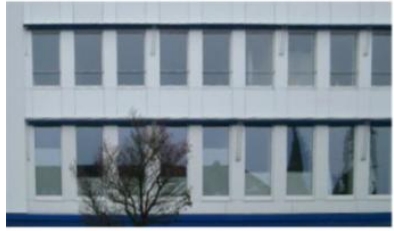

(d) Output(rank=77)

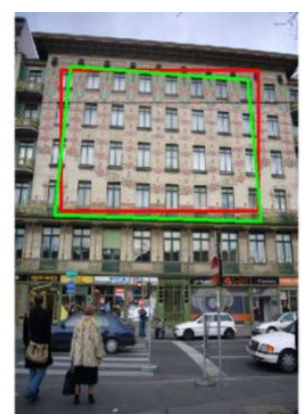

(b) Input(rank=77)

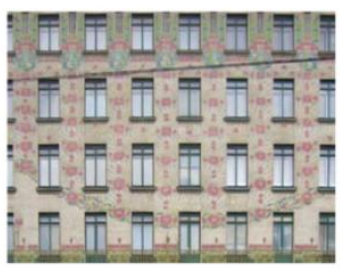

(e) Output(rank=61)

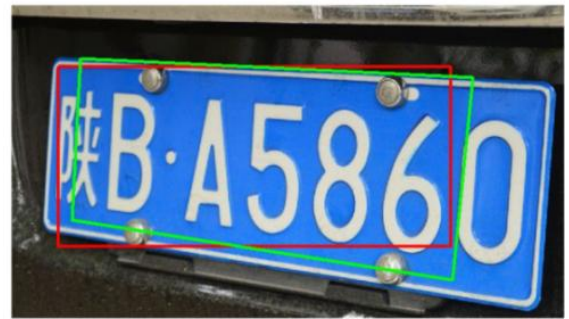

(c) Input(rank=64)

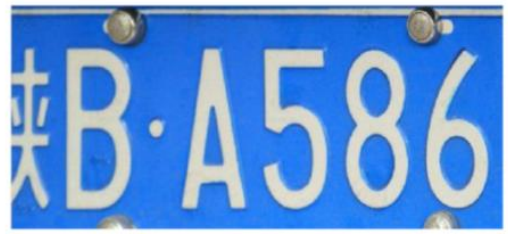

(f) Output(rank=52)

Fig. 1 Some results of TILT. (a)-(c): three deformed images containing "intrinsic" low-rank textures. The user-specified red windows in these images denote the regions to be processed by TILT, and the green window contains the deformed and corrupted low-rank images. (d)-(f): the recovered low-rank texture images from those green windows in (a)-(c), respectively.

\footnotetext{
${ }^{2}$ For example, if $H$ denotes a projective transformation in (1), then $(I \circ H)(x, y)$ may be computed as $I\left(\frac{h_{11} x+h_{12} y+h_{13}}{h_{31} x+h_{32} y+h_{33}}, \frac{h_{21} x+h_{22} y+h_{23}}{h_{31} x+h_{32} y+h_{33}}\right)$. ${ }^{3}$ Strictly speaking, as discussed in [33], $\nabla I$ is a 3D tensor: it gives a vector of derivatives at each pixel whose length is the number of the parameters in the transformation $H$. When $\nabla I$ is multiplied by another matrix or vector, it contracts in the obvious way which should be clear from the context.
} 
Fig. 1 illustrates some results of TILT. As shown in Fig. 1, the ranks of the image matrices for the recovered

textures are less than those of the image matrices for the deformed images. In addition to the recovered low-rank textures, the local distortion transformations between the deformed low-rank textures and their correspondingly recovered ones can also be obtained by using TILT. As shown in Fig. 1(d)-(f), the deformed low-rank textures will be approximately rectified to some new ones by these local transformations. Accordingly, a coarsely rectified image will also be obtained when the local transformation is applied to the whole image. Further, the rectified image resembles one captured by a front-viewpoint camera. Considering that, we will apply TILT to eliminate the projective distortion between images in the subsequently proposed matching method.

\section{Proposed image matching method}

When TILT is, respectively, performed on the reference image and the image to be matched, two rectified images are obtained. More important, the two rectified images seem to be captured by a front-viewpoint camera but with different parameters. Thus to a first approximation, there exists a similarity distortion (i.e., just containing scale and translation distortions) between them, and the projective distortion between the original input images is thus greatly eliminated. As a result, the problem of matching images with projective distortion reduces to a problem of matching images with similarity distortion to a first approximation.

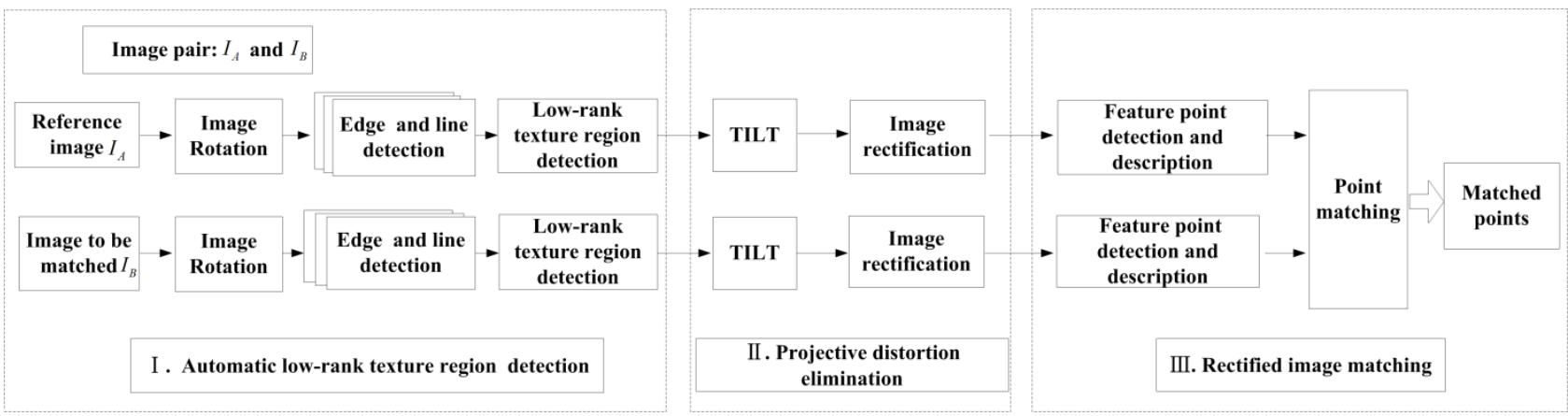

Fig. 2 Diagram of the proposed method.

Fig. 2 gives the diagram of the proposed algorithm, which mainly consists of the following three steps: First, a low-rank texture region detection algorithm is presented to automatically extract the low-rank texture regions from the reference image and the image to be matched. Secondly, the two extracted low-rank texture regions are, respectively, processed via TILT so that their ranks are minimum when viewed as matrices. As well, the local projective transformation matrices for the two input low-rank texture regions are coarsely estimated in this step. With the coarsely estimated transformation matrices, two newly produced images, as if they were captured by a front-viewpoint camera, are created from the original input images. Afterwards, there may exist translation and scale distortions between the two rectified images but with very little projective distortion. Thus the projective distortion between the two original input images is greatly reduced. Finally, a point-feature based matching method is performed on the two rectified images to obtain the final corresponding points.

In the following subsections, we will discuss each part in detail. 


\subsection{Automatic low-rank texture region detection}

As discussed in [33], TILT works effectively with low-rank texture regions. Therefore, a user-specified region that roughly contains the "intrinsic" low-rank textures should be manually selected from an image before it is fed into TILT. This is time-intensive and limits its application in many computer vision tasks, including image matching. Therefore, an automatic low-rank texture region detection method is first presented and then performed on the reference image and the image to be matched, respectively.

Many urban environments and man-made objects that contain a large number of regular structures or symmetric patterns typically lead to low-rank textures [33]. Examples include building facades and windows, words, and numbers. Moreover, these low-rank texture regions in the 3D scene often contain some parallel lines, and these lines generally correspond to the borders of the objects. Thus these parallel lines can be used to determine the borders of the low-rank texture regions. However, because of the projective distortion, these parallel lines in the 3D scene will no more be parallel in the $2 \mathrm{D}$ images. Therefore, in the proposed detection method, the input images are first rotated by some angles. Then a parallel line detector based on Canny edge detection [38] and Hough Transform [39] is performed on each rotated image. Finally, these parallel lines in different rotated images are jointly employed to determine a rectangle region, which roughly contains the "intrinsic" low-rank textures. The diagram of the proposed low-rank texture region detection is illustrated in Fig. 3 and its computation procedure is described as follows in detail.

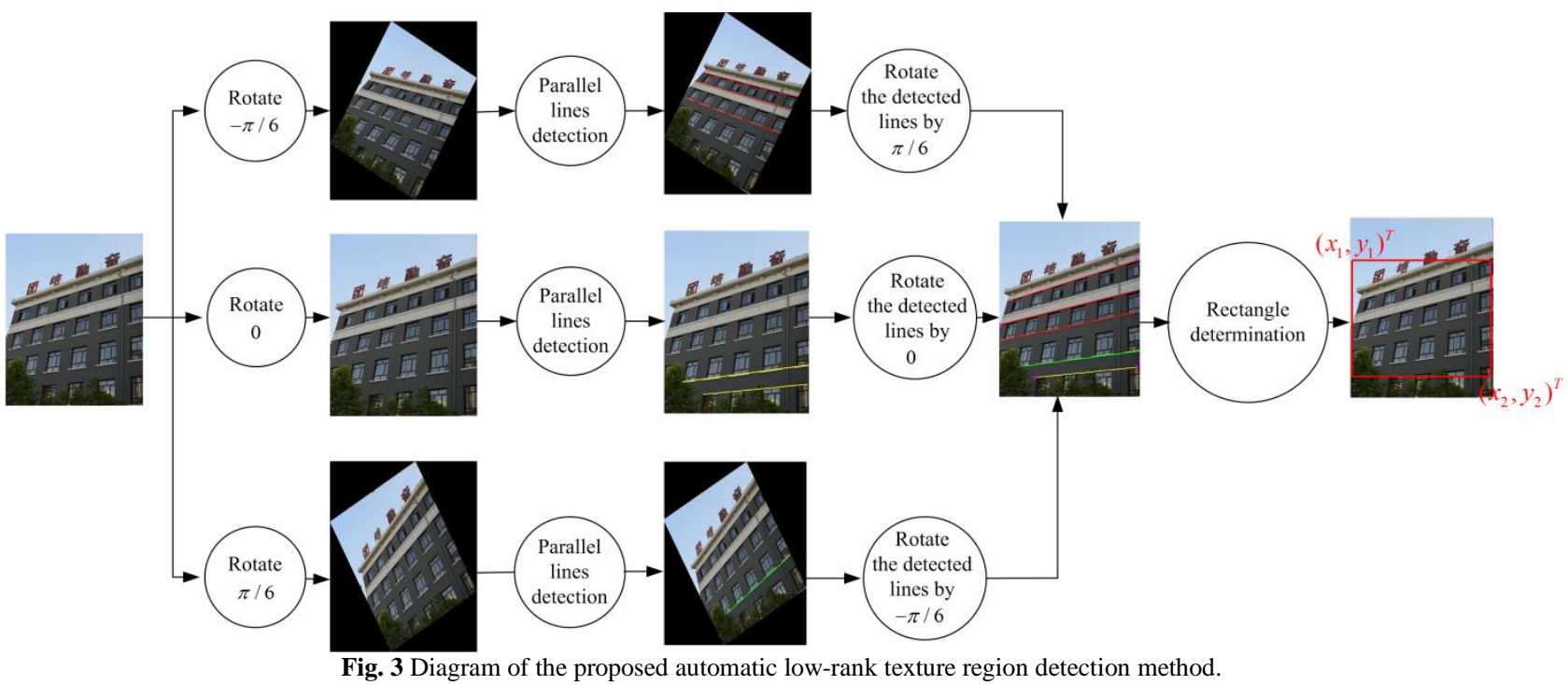

(1). Rotate the input image $I$ by $K$ different angles $\left\{\theta_{k} \mid k=1,2, \ldots, K\right\}$, respectively, and then obtain $K$ rotated images $\left\{I_{\theta_{k}} \mid k=1,2, \ldots, K\right\}$. In general, the more angles the input image is rotated by, the more parallel lines in the 3D scene will be detected and the more low-rank textures the detected region will contain. Unfortunately, this will increase the computation complexity. In this paper, we experimentally rotate the input image just by three angles, i.e., $\theta_{1}=-\pi / 6, \theta_{2}=0$ and $\theta_{3}=\pi / 6$.

(2). Perform the edge detection on each rotated image $I_{\theta_{k}}(k=1,2,3)$. Here, the Canny edge detector [38] is 
employed because of its accuracy and efficiency in detecting the true edges of an image. Of course, other edge detectors, such as Prewitt, Sobel or Roberts [39], can also be used.

(3). Perform the Hough transform [39] on the detected edges and obtain $n_{k}$ (approximately) parallel lines $\left\{\mathbf{l}_{\theta_{k}, i} \mid i=1,2, \ldots, n_{k}\right\}$ in each rotated image $I_{\theta_{k}}(k=1,2,3)$. Generally, a number of parallel lines in an image will lead to a local peak in the Hough transform domain. Accordingly, the highest peak in the Hough-transform domain corresponds to a set of parallel lines with the largest number in an image. Therefore, the direction that possesses the highest peak in the Hough-transform domain is selected as the main direction $\varsigma_{0}$, and the lines along the direction $\varsigma_{0}$ are selected. However, considering that the parallel lines in the 3D scene will generally not be exactly parallel in the deformed 2D images, the lines $\left\{\mathbf{l}_{\theta_{k}, i} \mid i=1,2, \ldots, n_{k}\right\}$, whose directions are in the range of $\left[\varsigma_{0}-\tau, \varsigma_{0}+\tau,\right]$, are all selected in order to detect more lines in each rotated image $I_{\theta_{k}}$. Here, $\tau$ is set to $\pi / 12$.

(4). Rotate the extracted lines $\left\{\mathbf{l}_{\theta_{k}, i} \mid i=1,2, \ldots, n_{k}\right\}$ in the image $I_{\theta_{k}}$ by an angle $-\theta_{k}$ and then put them back into the original input image $I$. Thus, as shown in Fig. 3, a total of $n_{K}=\sum_{k=1}^{K} n_{k}$ lines $\left\{\mathbf{l}_{i}^{\prime} \mid i=1,2, \ldots, n_{K}\right\}$ are obtained in the image $I$.

(5). Determine the low-rank texture region in the original input image using those detected lines $\left\{\mathbf{l}_{i}^{\prime} \mid i=1,2, \ldots, n_{K}\right\}$. Here, as in [33], the low-rank texture region is assumed to be indicated by a rectangle, which will be further determined by two points, i.e., the top-left vertex point $\mathbf{p}_{1}=\left(x_{1}, y_{1}\right)^{T}$ and the bottom-right one $\mathbf{p}_{2}=\left(x_{2}, y_{2}\right)^{T}$. Suppose each detected line $\mathbf{l}_{i}^{\prime}\left(i=1,2, \ldots, n_{K}\right)$ has two end-points $\mathbf{p}_{i 1}=\left(x_{i 1}, y_{i 1}\right)^{T}$ and $\mathbf{p}_{i 2}=\left(x_{i 2}, y_{i 2}\right)^{T}$. Thus, as shown in Fig. 3, the coordinates of the two vertex points are determined by

$$
\begin{aligned}
& x_{1}=\min _{i}\left(\min \left(x_{i, 1}, x_{i, 2}\right)\right), y_{1}=\min _{i}\left(\min \left(y_{i, 1}, y_{i, 2}\right)\right) \\
& x_{2}=\max _{i}\left(\max \left(x_{i, 1}, x_{i, 2}\right)\right), y_{2}=\max _{i}\left(\max \left(y_{i, 1}, y_{i, 2}\right)\right)
\end{aligned} .
$$
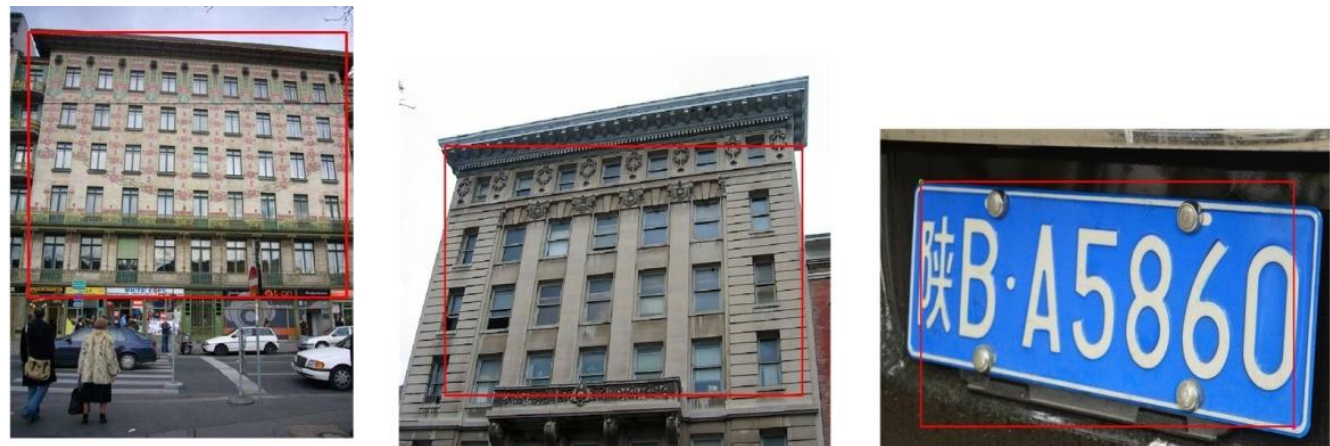

Fig. 4 Some examples of the low-rank textures detected by the proposed method. The detected red rectangles contain most of the "intrinsic" low-rank textures within these images.

The results of our proposed detection method on some low-rank texture images are illustrated in Fig. 4. It can be seen that the low-rank texture regions contained in these images are well detected by our proposed method. 


\subsection{Projective distortion elimination}

After the "intrinsic" low-rank texture regions in the reference image $I_{A}$ and the image to be matched $I_{B}$ are detected by the automatic low-rank texture region detection method described above, these texture regions are then fed into TILT and recovered by using (5) in Section 2. As discussed in Section 2, two local distortion transformations $H_{A}$ and $H_{B}$ between the deformed low-rank textures and their correspondingly recovered ones are also obtained by using TILT. When the local transformations $H_{A}$ and $H_{B}$ are performed on the images $I_{A}$ and $I_{B}$, two coarsely rectified images $I_{A}^{\prime}$ and $I_{B}^{\prime}$ are obtained, respectively. As shown in Fig. 5(b) and (d), the two rectified images are approximately close to two images captured by a camera from a front-viewpoint but with different parameters.

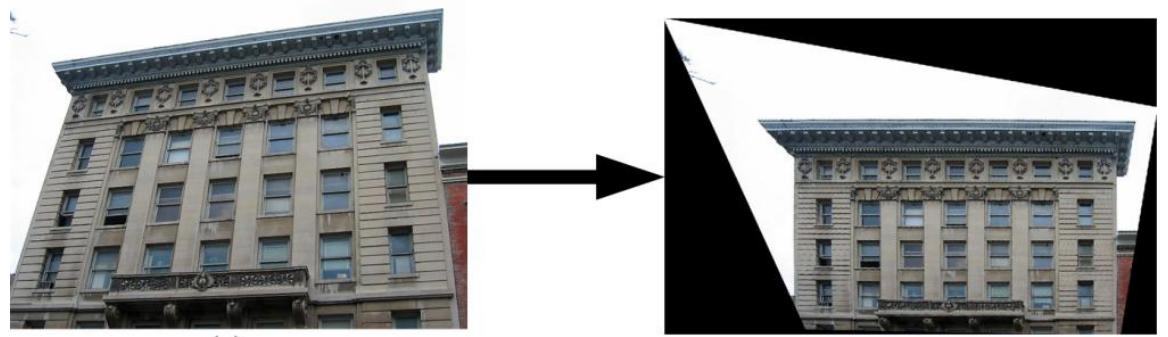

(a)

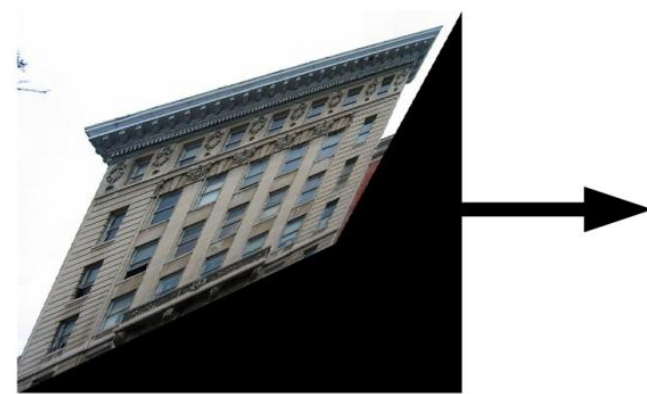

(c) (b)

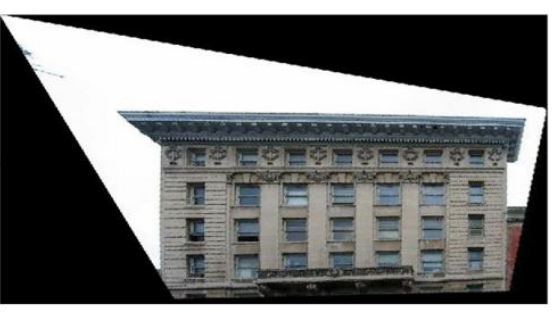

(d)

Fig. 5 Image rectification results on the reference image and the image to be matched. (a) reference image $I_{A}$; (b) rectified image $I_{A}^{\prime}$; (c) image to be matched $I_{B} ;(\mathrm{d})$ rectified image $I_{B}^{\prime}$.

\subsection{Rectified image matching}

Most of the existing matching methods could be employed to match the two rectified images. As one of the most representative descriptor methods, SIFT [19] has been widely applied to image matching. It works effectively for images with translation, scaling, and rotation distortions.

However, the two rectified images generally contain a large number of repetitive structures or patterns, and thus result in many similar local features. Correspondingly, many mismatches will be obtained if the original SIFT descriptor is simply employed in the matching of the two rectified images. For example, as shown in Fig. 6, both points $\mathbf{p}_{1}$ and $\mathbf{p}_{2}$ (with similar local information) will be matched by the same point $\mathbf{q}$ in another image. This may result from the fact that the original SIFT descriptors only contain their local information of these points.

As discussed in [40] the geometric shape information of the points in a large region around a point of interest could 
further improve its global distinctiveness. For example, as illustrated in Fig. 6, both $\mathbf{p}_{1}$ and $\mathbf{p}_{2}$ will be taken as the

correspondences of $\mathbf{q}$, if the local information of the points $\mathbf{p}_{1}, \mathbf{p}_{2}$ and $\mathbf{q}$ is only considered. However, if nonlocal information is considered, $\mathbf{p}_{1}$ will be still taken as the correspondence of $\mathbf{q}$, but $\mathbf{p}_{2}$ will be discarded. This is as a result of the following fact. The nonlocal information between the points $\mathbf{p}_{1}^{\prime}$ and $\mathbf{q}^{\prime}$ is more similar than that between the points $\mathbf{p}_{2}^{\prime}$ and $\mathbf{q}^{\prime}$.

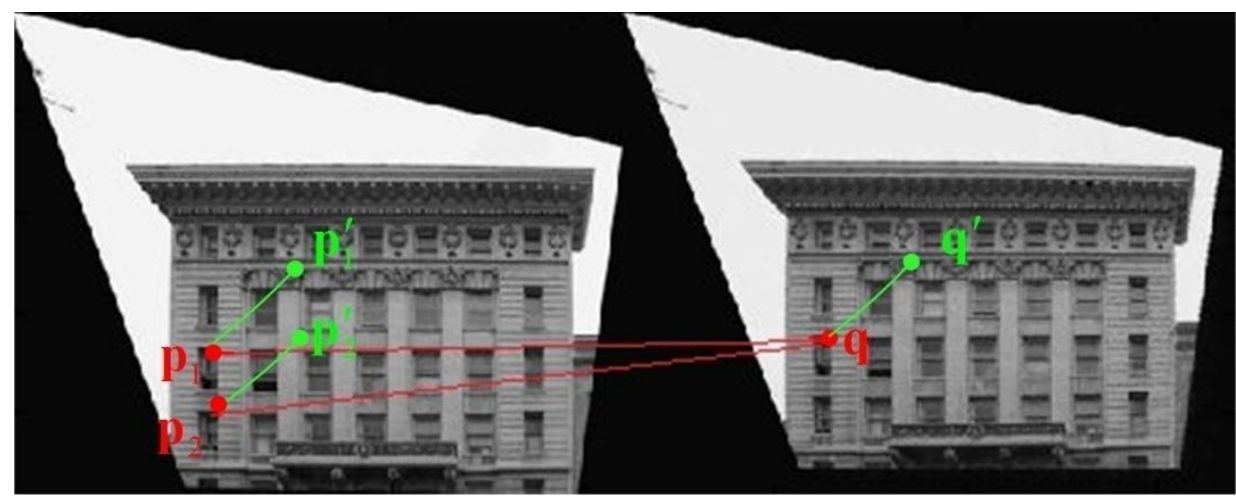

Fig. 6 An example of the mismatches obtained by SIFT. $\mathbf{q}$ is an interest point in one image. $\mathbf{p}_{1}$ and $\mathbf{p}_{2}$ are two similar points in another image, both of which are taken as the correspondences of the point $\mathbf{q}$ when considering the similarities among their SIFT descriptors. $\mathbf{p}_{1}^{\prime}, \mathbf{p}_{2}^{\prime}$ and $\mathbf{q}^{\prime}$ are three points that are far from the points $\mathbf{p}_{1}, \mathbf{p}_{2}$ and $\mathbf{q}$. The distances between $\mathbf{p}_{1}^{\prime}$ and $\mathbf{p}_{1}, \mathbf{p}_{2}^{\prime}$ and $\mathbf{p}_{2}, \mathbf{q}^{\prime}$ and $\mathbf{q}$ are all the same, and the shifts are all in the same direction.

Therefore, we will construct a new descriptor for each interest point by integrating a geometric shape descriptor $\mathbf{d}_{\text {shape }}$ with the traditional SIFT descriptor $\mathbf{d}_{\text {sift }}$. The SIFT descriptor $\mathbf{d}_{\text {sift }}$ contains the local information of each interest point, while the geometric shape descriptor $\mathbf{d}_{\text {shape }}$ contains the shape information of the points within a larger region (or even the whole image) around each interest point.

In the following steps, we will first describe the matching of the two rectified images briefly, and then describe the construction of the geometric shape descriptor $\mathbf{d}_{\text {shape }}$ in detail.

(1). Perform the Difference of Gaussian (DoG) interest point detection [19] on the two rectified images $I_{A}^{\prime}$ and $I_{B}^{\prime}$, respectively, and thus obtain two interest point sets.

(2). Construct the SIFT descriptor $\mathbf{d}_{\text {sift }}$ [19] as a 128-dimension vector for each interest point to describe its local information.

(3). Build the geometric shape descriptor $\mathbf{d}_{\text {shape }}$ as a 20-dimension vector for each interest point to describe the shape information of the pixels around each interest point. This step will be described in more detail later.

(4). Construct the final descriptor $\mathbf{d}$ by integrating the SIFT descriptor $\mathbf{d}_{\text {sift }}$ with the geometric shape descriptor $\mathbf{d}_{\text {shape }}$ as a 148-demension vector, i.e., 


$$
\mathbf{d}=\left[\begin{array}{c}
\omega \mathbf{d}_{\text {sift }} \\
(1-\omega) \mathbf{d}_{\text {shape }}
\end{array}\right],
$$

where $\omega$ is a weight and is simply set to 0.5 in this paper.

(5) Match the interest points in the two images $I_{A}^{\prime}$ and $I_{B}^{\prime}$ by using the nearest neighbor distance ratio method [41]. More specifically, given a point $\mathbf{p}_{A}$ in the image $I_{A}^{\prime}$, and its first and second nearest points $\mathbf{p}_{B 1}, \mathbf{p}_{B 2}$ in the image $I_{B}^{\prime}, \mathbf{p}_{A}$ and $\mathbf{p}_{B 1}$ are seen as a pair of matched points if they satisfy

$$
\frac{\left\|\mathbf{d}_{\mathbf{p}_{A}}-\mathbf{d}_{\mathbf{p}_{B 1}}\right\|_{2}}{\left\|\mathbf{d}_{\mathbf{p}_{A}}-\mathbf{d}_{\mathbf{p}_{B 2}}\right\|_{2}} \leq t h 1
$$

where $\|\cdot\|_{2}$ denotes the $l_{2}$-norm of a vector. $\mathbf{d}_{\mathbf{p}_{A}}, \mathbf{d}_{\mathbf{p}_{B 1}}$ and $\mathbf{d}_{\mathbf{p}_{B 2}}$ are the descriptors of the points $\mathbf{p}_{A}, \mathbf{p}_{B 1}$ and $\mathbf{p}_{B 2}$, respectively. And th 1 is a threshold and is experimentally set to 0.7 in this paper. The matched point pairs in the two rectified images $I_{A}^{\prime}$ and $I_{B}^{\prime}$ are also taken as the final matching results on the original input images $I_{A}$ and $I_{B}$.

Next we elaborate the construction of the geometric shape descriptor in Step (3).

Given a point $\mathbf{p}=(x, y)^{T}$ in an image $I$, its shape information can be indicated by its maximum curvature $\varphi(\mathbf{p})$ [40], which is computed as the maximum eigenvalue of its Hessian matrix, i.e.,

$$
\varphi(\mathbf{p})=\max \left(\lambda_{1}(\mathbf{p}), \lambda_{2}(\mathbf{p})\right)
$$

where $\lambda_{1}(\mathbf{p})$ and $\lambda_{2}(\mathbf{p})$ are two eigenvalues of the Hessian matrix $\Gamma(\mathbf{p})=\left[\begin{array}{cc}I_{x x}(\mathbf{p}) & I_{x y}(\mathbf{p}) \\ I_{x y}(\mathbf{p}) & I_{y y}(\mathbf{p})\end{array}\right]$. Further $I_{x x}(\mathbf{p})$ and $I_{y y}(\mathbf{p})$ are the second-order partial derivatives at the point $\mathbf{p}$ along $x$ - and $y$-directions, respectively. And $I_{x y}(\mathbf{p})$ is the corresponding second-order mixed derivative.

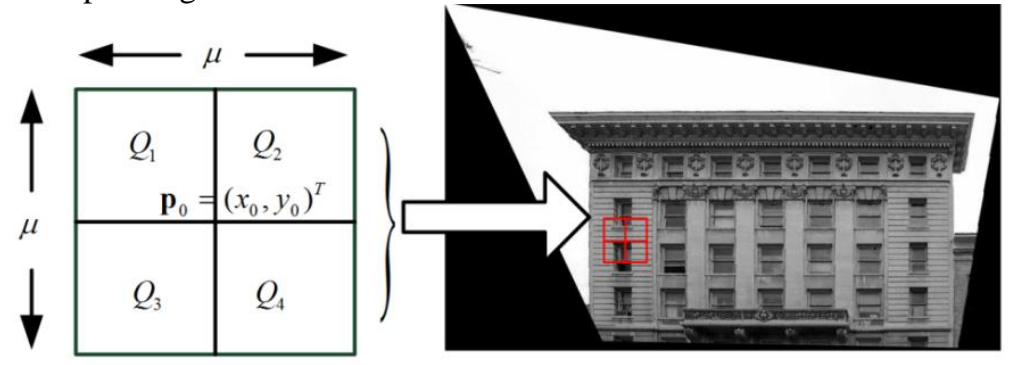

(a)

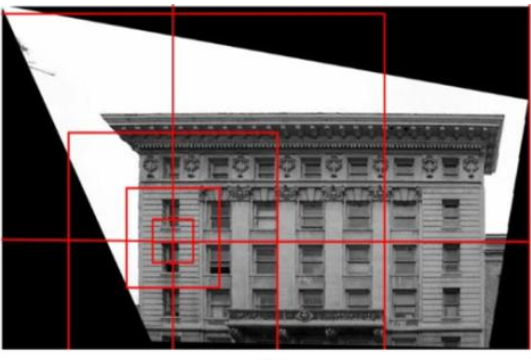

(b)

Fig. 7 Construction of the geometric shape descriptor $\mathbf{d}_{\text {shape }}$ for the interest point $\mathbf{p}_{0}=\left(x_{0}, y_{0}\right)^{T}$. (a) 4 sub-squares $\left\{Q_{i} \mid i=1,2,3,4\right\}$ around $\mathbf{p}_{0}$ are defined to compute its geometric shape descriptor $\mathbf{d}$ ; (b) 20 sub-squares around $\mathbf{p}_{0}$ are defined to compute its geometric shape descriptor $\mathbf{d}_{\text {shape }, 20}$.

Generally, the geometric shape descriptor for each interest point can be described as follows. As shown in Fig. 7(a), a square region of side length $\mu$ is first defined around each interest point $\mathbf{p}_{0}=\left(x_{0}, y_{0}\right)^{T}$ and then equally divided into 4 sub-squares $\left\{Q_{i} \mid i=1,2,3,4\right\}$ of side length $\frac{\mu}{2}$. Subsequently, a geometric shape descriptor $\mathbf{d}_{\text {shape }, 4}$ for the current point $\mathbf{p}_{0}$ is constructed as 


$$
\mathbf{d}_{\text {shape }, 4}=\left[\alpha_{Q_{1}}, \alpha_{Q_{2}}, \alpha_{Q_{3}}, \alpha_{Q_{4}}\right]^{T},
$$

where $\alpha_{Q_{i}}(i=1,2,3,4)$ denotes the curvature information of the points within the sub-square $Q_{i}$ and is defined as

$$
\alpha_{Q_{i}}=\sum_{\mathbf{p} \in Q_{i}} \varphi(\mathbf{p})
$$

However, some similar local feature points might still be not well distinguished if only a square (or 4 sub-squares) of fixed side length is considered. For example, as shown in the left image of Fig. 6, the curvature information of the pixels around the interest points $\mathbf{p}_{1}$ and $\mathbf{p}_{2}$ would be still of great similarity if the side length of the square is set to too short. Similar phenomenon would also occur when the side length is set to too long. Therefore, centered on each interest point, a set of squares $\left\{S_{j} \mid j=1,2, \ldots, n\right\}$ of different side lengths, instead of one square, are defined to improve the distinctiveness and robustness of the descriptor. As shown in Fig. 7(b), each square $\left\{S_{j} \mid j=1,2, \ldots, n\right\}$ is further divided into four sub-squares, and thus we obtain a set of sub-squares $\left\{Q_{j, i} \mid j=1,2, \ldots, n ; i=1,2,3,4\right\}$. Here, similar to that in [40], 5 squares (i.e., $n$ is set to 5) are used and the side length of the $j$-th square $S_{j}$ is set to $\frac{v}{2^{j-1}}$, where $v$ is the larger between the height and the width of the image. Then the geometric shape descriptor is described as a 20-dimension vector, i.e.,

$$
\mathbf{d}_{\text {shape }, 20}=\left[\alpha_{Q_{1,1}}, \alpha_{Q_{1,2}}, \alpha_{Q_{1,3}}, \alpha_{Q_{1,4}}, \ldots, \alpha_{Q_{5,1}}, \alpha_{Q_{5,2}}, \alpha_{Q_{5,3}}, \alpha_{Q_{5,4}}\right]^{T},
$$

where $\alpha_{Q_{j, i}}(j=1,2,3,4,5 ; i=1,2,3,4)$ denotes the curvature information of the pixels within the sub-square $Q_{j, i}$, and is computed similar to (11). After being normalized, the final geometric shape descriptor $\mathbf{d}_{\text {shape }}$ is constructed as

$$
\mathbf{d}_{\text {shape }}=\frac{\mathbf{d}_{\text {shape }, 20}}{\left\|\mathbf{d}_{\text {shape }, 20}\right\|_{2}} .
$$

Fig. 8 illustrates the matching results on a pair of rectified images by using the proposed descriptor. It demonstrates that the introduction of the geometric shape information of the pixels around each considered point

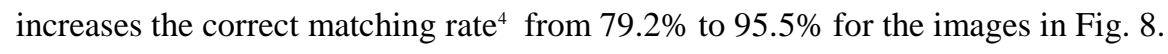

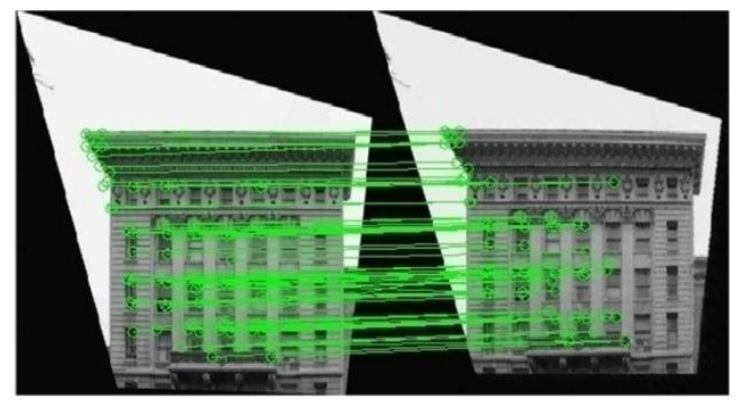

(a)

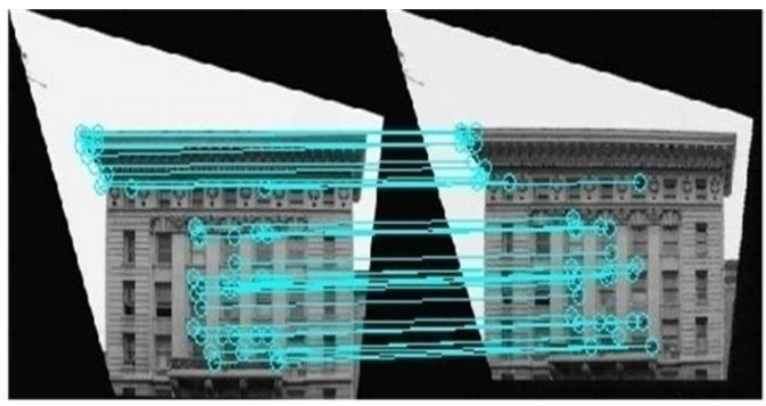

(b)

Fig. 8 Illustration of the matching results on a pair of rectified images by using the proposed descriptor. (a) the result (correct matching rate is 61/77) obtained by only using the traditional SIFT descriptor, i.e., $\omega$ is set to 1 in (7). Here, the number 61 denotes the correct matches and the number 77 denotes the total matches. (b) the result (correct matching rate is 43/45) obtained by jointly using the geometric shape descriptor and the traditional SIFT descriptor, i.e., $\omega$ is set to 0.5 in (7). Similarly, the numbers 43 and 45 denote the correct and total matches, respectively.

\footnotetext{
${ }^{4}$ The correct matching rate is defined as the ratio between the numbers of correct and total matches. See (15) in Section 4.
} 


\section{Experiments}

In this section, three sets of experiments are performed to verify the validity of the proposed matching algorithm for images with projective distortion. A set of man-made images deformed by different projective transformations are first employed in Section 4.1. Several pairs of real-world images with small or large projective distortions are further employed to demonstrate the validity of the proposed method in Section 4.2 and Section 4.3, respectively. All the experiments were implemented on a personal computer with a $2.83 \mathrm{GHz}$ Intel Core CPU, 3.84G RAM, and Windows 7 operation system.

In addition to the proposed one(s), another three matching algorithms are employed for comparisons, which include the SIFT [19], MSER [21] and ASIFT [20] methods. The number of correct matches $\left(N_{C}\right)$, the feature repeatability rate $\left(F_{R}\right)$ of detected features and the correct matching rate $\left(C_{R}\right)$ are employed to evaluate different matching methods [14]. Here, given the estimated projective transformation matrix ${ }^{5} H$ in Eq. (1), a matched point pair $\left\{\mathbf{p}_{A}=(x, y)^{T}, \mathbf{p}_{B}=\left(x^{\prime}, y^{\prime}\right)^{T}\right\}$ is deemed as a correct one if they satisfy

$$
\sqrt{\left(x-x^{\prime}\right)^{2}+\left(y-y^{\prime}\right)^{2}}<\operatorname{th} 2, \quad x^{\prime}=\frac{h_{11} x+h_{12} y+h_{13}}{h_{31} x+h_{32} y+h_{33}}, y^{\prime}=\frac{h_{21} x+h_{22} y+h_{23}}{h_{31} x+h_{32} y+h_{33}},
$$

where th 2 is a threshold and is experimentally set to 5 in all of the experiments. $C_{R}$ is defined as the ratio between the numbers of correct matches $N_{C}$ and total matches $N_{T}$, i.e.,

$$
C_{R}=N_{C} / N_{T} \text {. }
$$

The quantity $F_{R}$ is defined as the ratio of the number of correct matches to the minimum number of the total detected feature points from the two input images [14], i.e.,

$$
F_{R}=N_{C} / \min \left(N_{r e f}, N_{\text {test }}\right) \text {, }
$$

where $N_{\text {ref }}$ and $N_{\text {test }}$ denote the total number of the detected feature points from the reference image and the image to be matched, respectively.

\subsection{Matching man-made images with projective distortions}

In this subsection, a set of man-made test images with projective distortions are employed to verify our proposed method(s). Fig. 9 illustrates the test images, in which, Fig. $9(a)^{6}$ is taken as the reference image in the following experiments. Fig. 9(b)-(h) are the images to be matched, which are obtained by performing the projective transformations $H=\left[\begin{array}{ccc}1 & 0 & 0 \\ 0 & 1 & 0 \\ \beta & 0.0002 & 1\end{array}\right]$ on Fig. 9(a) with $\beta=0.0002,0.0004,0.0006,0.0008,0.001,0.0015$, and 0.002 respectively.

\footnotetext{
${ }^{5}$ The projective transformation is estimated by performing the RANSAC algorithm [17] on the matched point pairs in this paper. ${ }^{6}$ The image is downloaded from http://perception.csl.illinois.edu/matrix-rank/tilt.html.
} 


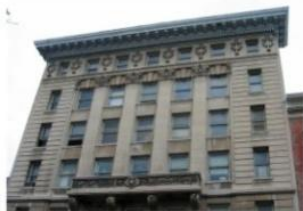

(a)

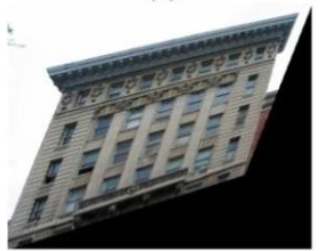

(e)

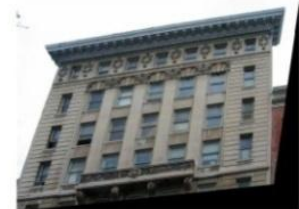

(b)

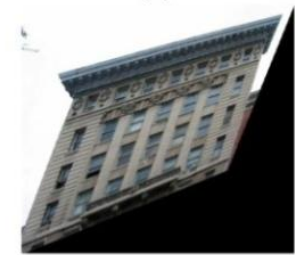

(f)

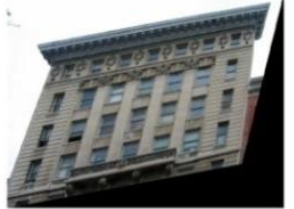

(c)

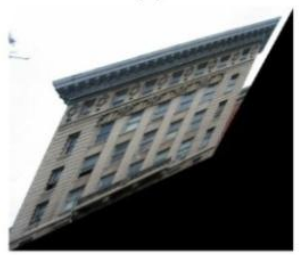

(g)

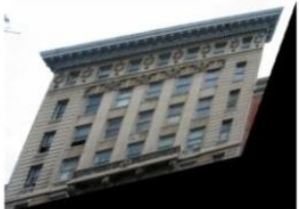

(d)

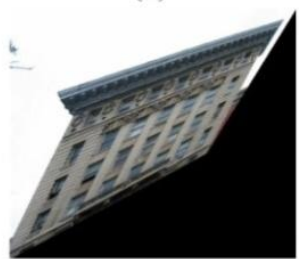

(h)

Fig. 9 A set of man-made images with projective distortions. (a) Reference image; (b) - (h) Test images derived from the reference image in (a) with different projective distortions.

Table 1 Matching results of different methods on the man-made images with projective distortions.

\begin{tabular}{|c|c|c|c|c|c|c|}
\hline Test images & Metric & SIFT & MSER & ASIFT & Proposed $(\omega=1)$ & Proposed $(\omega=0.5)$ \\
\hline \multirow{3}{*}{ Fig.9(b) } & $N_{C} / N_{T} / \operatorname{Min}\left(N_{r e f}, N_{t e s t}\right)$ & $50 / 58 / 143$ & $106 / 149 / 487$ & $3613 / 4060 / 33260$ & $80 / 88 / 132$ & $74 / 76 / 132$ \\
\hline & $C_{R}$ & $86.21 \%$ & $71.44 \%$ & $88.9 \%$ & $90.91 \%$ & $97.37 \%$ \\
\hline & $F_{R}$ & $34.97 \%$ & $21.77 \%$ & $10.86 \%$ & $60.61 \%$ & $56.06 \%$ \\
\hline \multirow{3}{*}{ Fig.9(c) } & $N_{C} / N_{T} / \operatorname{Min}\left(N_{r e f}, N_{\text {test }}\right)$ & $42 / 50 / 147$ & $90 / 106 / 463$ & $1808 / 2021 / 31481$ & $83 / 92 / 132$ & $82 / 84 / 132$ \\
\hline & $C_{R}$ & $84 \%$ & $84.91 \%$ & $89.64 \%$ & $90.22 \%$ & $97.62 \%$ \\
\hline & $F_{R}$ & $28.57 \%$ & $19.44 \%$ & $5.75 \%$ & $62.88 \%$ & $62.12 \%$ \\
\hline \multirow{3}{*}{ Fig.9(d) } & $N_{C} / N_{T} / \operatorname{Min}\left(N_{\text {ref }}, N_{\text {test }}\right)$ & $30 / 39 / 139$ & $71 / 85 / 416$ & $1214 / 1424 / 29500$ & $58 / 67 / 132$ & $56 / 58 / 132$ \\
\hline & $C_{R}$ & $76.92 \%$ & $83 \%$ & $85.25 \%$ & $86.57 \%$ & $96.55 \%$ \\
\hline & $F_{R}$ & $21.58 \%$ & $17.07 \%$ & $4.12 \%$ & $43.94 \%$ & $42.42 \%$ \\
\hline \multirow{3}{*}{ Fig.9(e) } & $N_{C} / N_{T} / \operatorname{Min}\left(N_{r e f}, N_{t e s t}\right)$ & $10 / 20 / 145$ & $62 / 87 / 375$ & $977 / 1223 / 27180$ & $62 / 72 / 132$ & $46 / 48 / 132$ \\
\hline & $C_{R}$ & $50 \%$ & $71.26 \%$ & $79.89 \%$ & $86.11 \%$ & $95.83 \%$ \\
\hline & $F_{R}$ & $6.9 \%$ & $16.53 \%$ & $3.59 \%$ & $46.97 \%$ & $34.85 \%$ \\
\hline \multirow{3}{*}{ Fig.9(f) } & $N_{C} / N_{T} / \operatorname{Min}\left(N_{r e f}, N_{t e s t}\right)$ & $10 / 28 / 157$ & $38 / 67 / 357$ & $864 / 1025 / 26029$ & $65 / 73 / 131$ & $54 / 56 / 131$ \\
\hline & $C_{R}$ & $35.71 \%$ & $56.72 \%$ & $84.29 \%$ & $89.91 \%$ & $96.42 \%$ \\
\hline & $F_{R}$ & $6.37 \%$ & $10.64 \%$ & $3.32 \%$ & $49.62 \%$ & $40.91 \%$ \\
\hline \multirow{3}{*}{ Fig.9(g) } & $N_{C} / N_{T} / \operatorname{Min}\left(N_{r e f}, N_{\text {test }}\right)$ & $0 / 12 / 188$ & $40 / 54 / 292$ & $561 / 776 / 22557$ & $63 / 78 / 132$ & $53 / 54 / 132$ \\
\hline & $C_{R}$ & 0 & $74.07 \%$ & $72.29 \%$ & $80.77 \%$ & $98.18 \%$ \\
\hline & $F_{R}$ & 0 & $13.7 \%$ & $2.49 \%$ & $47.73 \%$ & $40.15 \%$ \\
\hline \multirow{3}{*}{ Fig.9(h) } & $N_{C} / N_{T} / \operatorname{Min}\left(N_{r e f}, N_{t e s t}\right)$ & 0/6/196 & $40 / 58 / 286$ & $428 / 614 / 19629$ & $61 / 77 / 132$ & $47 / 50 / 132$ \\
\hline & $C_{R}$ & 0 & $68.97 \%$ & $69.71 \%$ & $79.22 \%$ & $94 \%$ \\
\hline & $F_{R}$ & 0 & $13.99 \%$ & $2.18 \%$ & $46.21 \%$ & $35.6 \%$ \\
\hline
\end{tabular}

Table 1 gives the experimental results of different methods, which demonstrates that both the proposed method with $^{7} \omega=1$ and the proposed one with $\omega=0.5$ perform better than the others. In terms of the metrics $C_{R}$ and $F_{R}$, both of our proposed methods perform better than SIFT, MSER and ASIFT in all of the seven experiments. With the increase of $\beta$, our proposed methods show increased superiority. SIFT performs the worst among all of the methods.

\footnotetext{
${ }^{7}$ In the proposed method with $\omega=1$, the parameter $\omega$ in (7) is set to 1, i.e., only the SIFT descriptor is considered. Similarly, in the proposed method with $\omega=0.5$, the parameter $\omega$ in (7) is set to 0.5 , i.e., the proposed geometric shape descriptor and the local SIFT descriptor are jointly considered.
} 
It obtains the lowest correct matching rate in most cases. It even produces no matches when the projective distortion is

too large (e.g., $\beta$ is 0.0015 or 0.002 ). This may result from the fact that SIFT is only scale invariant and is sensitive to affine or projective distortion. ASIFT obtains the largest number of correct matches among the mentioned methods, but it also obtains the lowest repeatability in most cases. This may suggest that there are only a few feature points detected by ASIFT which are useful while many more are useless. Finally, it can also be found that the proposed method with $\omega=0.5$ performs better than the proposed one with $\omega=1$ in terms of the metric $C_{R}$. This owes to the introduction of the geometric shape descriptor when the rectified images are matched.

\subsection{Matching real-world images with small projective distortions}

In this subsection, four pairs of real-world images with small projective distortions are matched, which are displayed in Fig. $10^{8}$. The experimental results are provided in Table 2, which demonstrates that our proposed methods (with $\omega=1$ and $\omega=0.5$ ) perform competitively with the traditional SIFT and ASIFT methods and better than the MSER method in terms of the correct match rate for the images in Fig. 10. This may be as a result of the fact that the projective distortions between the input images in Fig. 10 could be approximated by a similarity or affine transformation. SIFT and ASIFT could be directly applied for these test images without the rectification. It is also found that the proposed method with $\omega=0.5$ performs better than the traditional SIFT method and the proposed method with $\omega=1$. This further demonstrates the validity of the shape descriptor introduced in Subsection 3.3. Similar to those in Table 1, ASIFT still obtains the lowest repeatability rate for most of the test images although it obtains the largest number of correct matches.

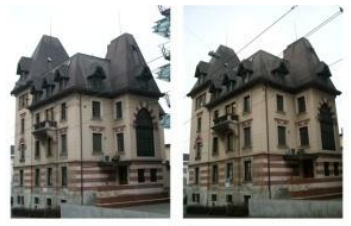

(a)

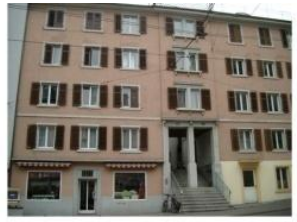

(b)

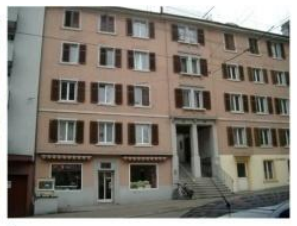

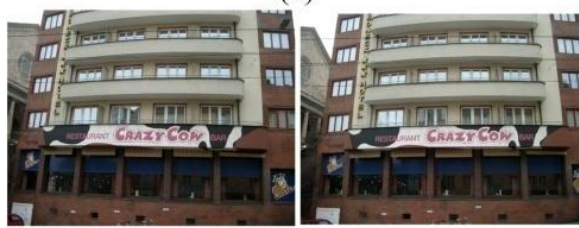

(c)

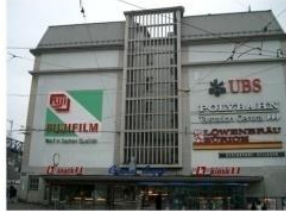

(d)

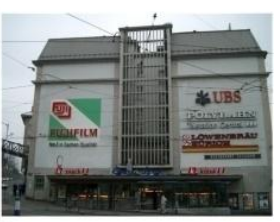

Fig. 10 Real-world images with small projective distortions. In (a)-(d), the left image is the reference one and the right image is the one to be matched.

Table 2 Matching results of different methods on the real-world images with small projective distortions.

\begin{tabular}{|c|c|c|c|c|c|c|}
\hline Test images & Metric & SIFT & MSER & ASIFT & Proposed $(\omega=1)$ & Proposed $(\omega=0.5)$ \\
\hline \multirow{3}{*}{ Fig.10(a) } & $N_{C} / N_{T} / \operatorname{Min}\left(N_{r e f}, N_{t e s t}\right)$ & $48 / 49 / 247$ & $40 / 45 / 154$ & $2447 / 2448 / 24741$ & $50 / 52 / 219$ & $52 / 52 / 219$ \\
\hline & $C_{R}$ & $97.96 \%$ & $88.89 \%$ & $99.96 \%$ & $96.15 \%$ & $100.00 \%$ \\
\hline & $F_{R}$ & $19.43 \%$ & $25.97 \%$ & $9.89 \%$ & $22.83 \%$ & $23.74 \%$ \\
\hline \multirow{2}{*}{ Fig.10(b) } & $N_{C} / N_{T} / \operatorname{Min}\left(N_{r e f}, N_{t e s t}\right)$ & $102 / 108 / 355$ & $71 / 120 / 496$ & $1724 / 1850 / 28988$ & $56 / 59 / 307$ & $35 / 35 / 307$ \\
\hline & $C_{R}$ & $94.44 \%$ & $59.17 \%$ & $93.19 \%$ & $94.92 \%$ & $100.00 \%$ \\
\hline
\end{tabular}

${ }^{8}$ These test images are downloaded from http://www.vision.ee.ethz.ch/showroom/zubud/index.en.html. 


\begin{tabular}{|c|c|c|c|c|c|c|}
\hline & $F_{R}$ & $28.73 \%$ & $14.31 \%$ & $5.95 \%$ & $18.24 \%$ & $11.40 \%$ \\
\hline \multirow{3}{*}{ Fig.10(c) } & $N_{C} / N_{T} / \operatorname{Min}\left(N_{r e f}, N_{t e s t}\right)$ & $123 / 145 / 252$ & $50 / 114 / 392$ & $2710 / 3046 / 25951$ & $116 / 141 / 227$ & $88 / 91 / 227$ \\
\hline & $C_{R}$ & $84.83 \%$ & $43.86 \%$ & $88.97 \%$ & $82.27 \%$ & $96.70 \%$ \\
\hline & $F_{R}$ & $48.81 \%$ & $12.76 \%$ & $10.44 \%$ & $51.10 \%$ & $38.77 \%$ \\
\hline \multirow{3}{*}{ Fig.10(d) } & $N_{C} / N_{T} / \operatorname{Min}\left(N_{r e f}, N_{t e s t}\right)$ & $30 / 34 / 110$ & $55 / 110 / 455$ & $3001 / 3189 / 29346$ & $37 / 42 / 107$ & $38 / 41 / 107$ \\
\hline & $C_{R}$ & $88.24 \%$ & $50.00 \%$ & $94.10 \%$ & $88.10 \%$ & $92.68 \%$ \\
\hline & $F_{R}$ & $27.27 \%$ & $8.87 \%$ & $10.23 \%$ & $34.58 \%$ & $35.51 \%$ \\
\hline
\end{tabular}

\subsection{Matching real-world images with larger relative projective distortions}

Sixteen pairs of real-world images with larger relative projective distortions are matched to verify the validity of our proposed method(s) in this subsection. Fig. 11 displays the test images and Fig. 12 illustrates the matched results of different methods on the test images in Fig. 11(4) ${ }^{9}$. The quantitative detection results of different methods are provided in Fig. 13 and Table 3.

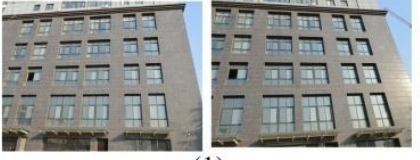

(1)

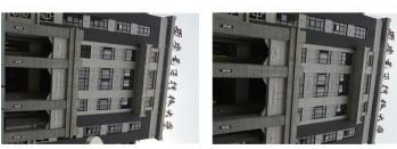

$(5)$

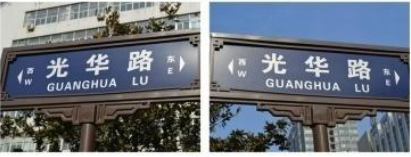

(9)

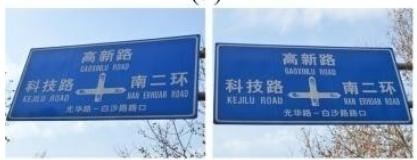

(13)

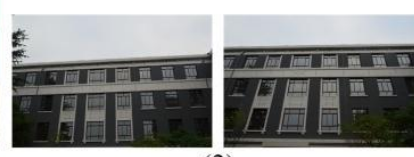
(2)
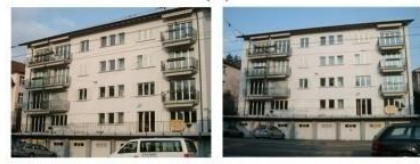

(6)

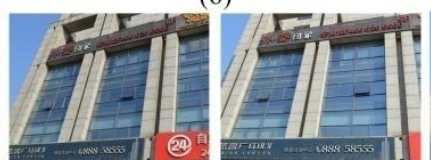

(10)

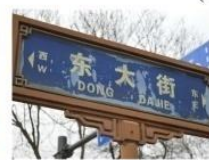

(14)
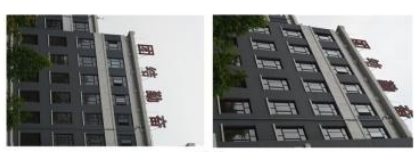
(3)
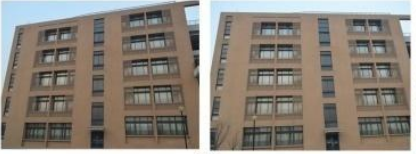

(7)

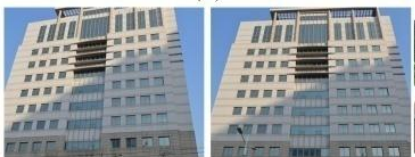

(11)

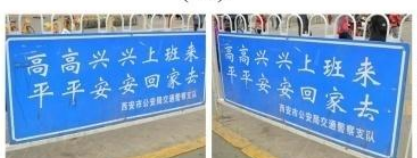

(15)

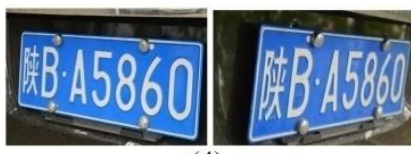

(4)

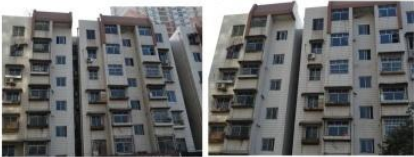

(8)

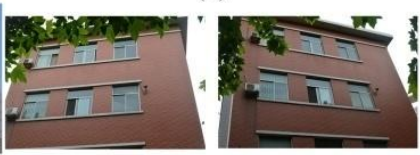

(12)

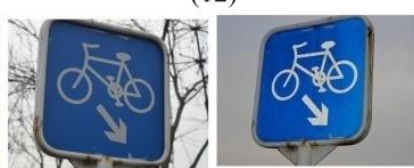

(16)

Fig. 11 Real-world test images with relative larger projective distortions. In (1)-(16), the left image is the reference one and the right image is the one to be matched.

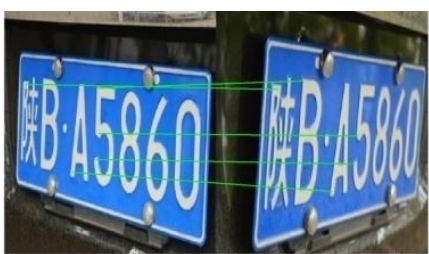

(a) $\operatorname{SIFT}\left(C_{R}=4 / 5=80 \%\right)$

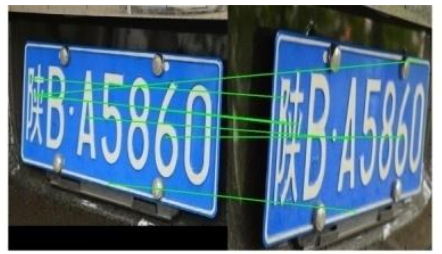

(b) $\operatorname{MSER}\left(C_{R}=5 / 7=71.43 \%\right)$

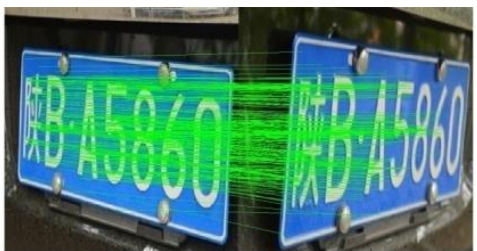

(c) $\operatorname{ASIFT}\left(C_{R}=268 / 288=93.06 \%\right)$
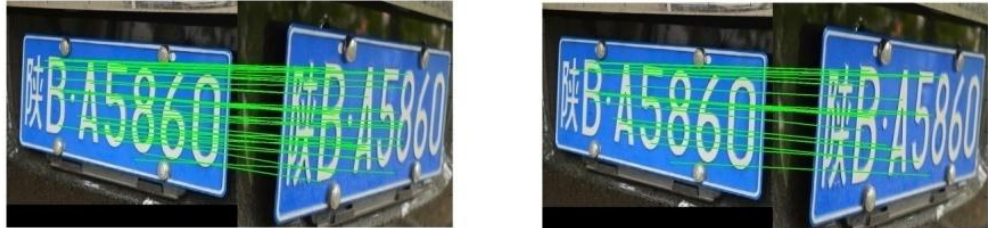

(d) Proposed $\omega=1\left(C_{R}=37 / 38=97.39 \%\right) \quad$ (e) Proposed $\omega=0.5\left(C_{R}=23 / 23=100 \%\right)$

Fig. 12 Matching results for the test images in Fig. 11(4).

As illustrated in Fig. 12, our proposed methods (with $\omega=1$ and $\omega=0.5$ ) and ASIFT perform better than SIFT

${ }^{9}$ Similar results are obtained for the other pairs of test images. Due to the space limitations, their results are not displayed here. 
and MSER in terms of the numbers of total and correct matches. It can also be found that our proposed methods obtain

higher correct matching rates than ASIFT. More specially, the correct matching rate of the proposed method with $\omega=0.5$ even achieves $100 \%$ for the test images in Fig. 11(4).

The experimental data in Fig. 13 further demonstrates the superiority of our proposed methods. In terms of the metric $C_{R}$, the proposed method with $\omega=0.5$ performs the best for most of test images. The proposed method with $\omega=1$ and ASIFT perform competitively and better than SIFT and MSER. Similar to what was discussed in Subsection 4.1, for some test images, such as the last five pairs of test images in Fig. 11, SIFT fails and obtains no correct matches. In terms of the metric $F_{R}$, the proposed methods with $\omega=1$ and $\omega=0.5$ perform better than the other methods, especially than SIFT and ASIFT. As shown in Fig. 13(b) and Table 3, ASIFT still obtains the largest number of correct matches but the lowest repeatability rates for most of the test images.

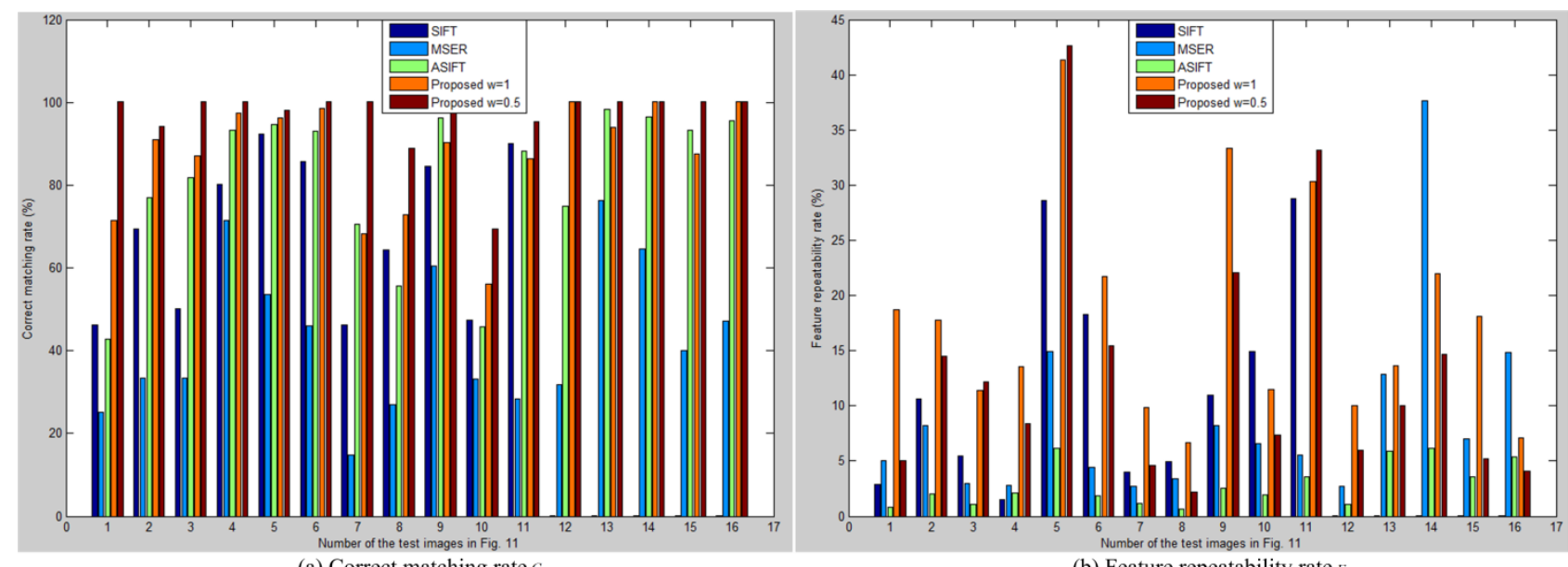

(a) Correct matching rate $C_{R}$

(b) Feature repeatability rate $F_{p}$

Fig. 13 Values of the metrics $C_{R}$ and $F_{R}$ obtained by different methods on the real-world images with large projective distortions.

Table 3 Number of Matches $N_{C} / N_{T} / \operatorname{Min}\left(N_{r e f}, N_{\text {test }}\right)$ obtained by different methods on the real-world images with large projective distortions.

\begin{tabular}{|c|c|c|c|c|c|}
\hline Test images & SIFT & MSER & ASIFT & Proposed $(\omega=1)$ & Proposed $(\omega=0.5)$ \\
\hline Fig.11(1) & $6 / 13 / 211$ & $38 / 116 / 574$ & $190 / 444 / 23686$ & $45 / 63 / 240$ & $12 / 12 / 240$ \\
\hline Fig.11(2) & $9 / 13 / 85$ & $21 / 63 / 256$ & $354 / 461 / 17236$ & $20 / 22 / 118$ & $16 / 17 / 118$ \\
\hline Fig.11(3) & $7 / 14 / 128$ & $8 / 24 / 272$ & $188 / 230 / 18016$ & $20 / 23 / 140$ & $17 / 17 / 140$ \\
\hline Fig.11(4) & $4 / 5 / 272$ & $5 / 7 / 178$ & $268 / 288 / 12667$ & $37 / 38 / 273$ & $23 / 23 / 273$ \\
\hline Fig.11(5) & $48 / 52 / 168$ & $54 / 101 / 361$ & $1405 / 1486 / 22755$ & $99 / 103 / 239$ & $99 / 101 / 232$ \\
\hline Fig.11(6) & $48 / 56 / 262$ & $17 / 37 / 387$ & $521 / 561 / 28516$ & $122 / 124 / 562$ & $87 / 87 / 562$ \\
\hline Fig.11(7) & $6 / 13 / 151$ & $16 / 109 / 591$ & $269 / 382 / 22579$ & $15 / 22 / 152$ & $7 / 7 / 152$ \\
\hline Fig.11(8) & $9 / 14 / 183$ & $15 / 56 / 447$ & $204 / 367 / 31586$ & $24 / 33 / 358$ & $8 / 9 / 358$ \\
\hline Fig.11(9) & $27 / 32 / 247$ & $32 / 53 / 391$ & $566 / 588 / 22386$ & $65 / 72 / 195$ & $43 / 43 / 195$ \\
\hline Fig.11(10) & $17 / 36 / 114$ & $44 / 133 / 667$ & $424 / 930 / 22054$ & $14 / 25 / 122$ & $9 / 13 / 122$ \\
\hline Fig.11(11) & $142 / 158 / 493$ & $31 / 110 / 559$ & $913 / 1035 / 25743$ & $216 / 250 / 712$ & $236 / 248 / 712$ \\
\hline Fig.11(12) & $0 / 6 / 71$ & $6 / 19 / 218$ & $148 / 198 / 13694$ & $10 / 10 / 100$ & $6 / 6 / 100$ \\
\hline Fig.11(13) & $0 / 3 / 40$ & $48 / 63 / 373$ & $1052 / 1070 / 17979$ & $15 / 16 / 110$ & $11 / 11 / 110$ \\
\hline Fig.11(14) & $0 / 3 / 165$ & $47 / 73 / 125$ & $1122 / 1163 / 18178$ & $27 / 27 / 123$ & $18 / 18 / 123$ \\
\hline Fig.11(15) & 0/1/191 & $30 / 75 / 426$ & $831 / 893 / 23187$ & $42 / 48 / 232$ & $12 / 12 / 232$ \\
\hline Fig.11(16) & $0 / 3 / 226$ & $8 / 17 / 54$ & $491 / 514 / 9200$ & $14 / 14 / 197$ & $8 / 8 / 197$ \\
\hline
\end{tabular}

In addition, the average computation time of different methods on the test images in Fig. 11 are provided in Table 
4. It is found that the higher performance of the proposed methods, especially the proposed method with $\omega=0.5$, is at

Table 4 Average computation time of different methods.

\begin{tabular}{cccccc}
\hline Methods & SIFT & MSER & ASIFT & Proposed $(\omega=1)$ & Proposed $(\omega=0.5)$ \\
\hline Time (in seconds) & 3.6058 & 6.5528 & 52.2357 & 28.7783 & 205.0950 \\
\hline
\end{tabular}

\section{Conclusion}

In this paper, a TILT based image matching method is proposed for low-rank texture images with projective distortion. By virtue of TILT, the problem of matching images with projective distortion reduces to a problem of matching two rectified images just with translation and scale distortions. Experimental results demonstrate the validity of the proposed method. For images with small projective distortions, which may be approximated by a similarity or affine transformation to some extent, the proposed method performs competitively and even slightly better than the traditional SIFT and ASIFT methods. For the images with larger relative projective distortions, the proposed method is shown to be obviously superior to the traditional SIFT, MSER and ASIFT methods.

In addition to the local information, some geometric shape information of the pixels within a larger region (or even the whole image) around each interest point is employed when the two rectified images are matched. This further improves the correct matching rate of the proposed method, especially for the images with many repetitive patterns or symmetric structures. Finally, an automatic low-rank texture region detection method is presented, by which, the low-rank textures are automatically selected from the original input images before they are fed into TILT. This makes the proposed method more applicable in practice.

\section{Acknowledgement}

This work of the first two and the last authors is supported by the National Natural Science Foundation of China under Grant No.61104212 and by the Fundamental Research Funds for the Central Universities under Grant No. NSIY211416. The work of the third author is supported by the National Science Foundation under Grant No. ECCS-1405579.

\section{References}

[1] P. Podbreznik, B. Potocnik, Estimating correspondence between arbitrarily selected points in two widely-separated views, Advanced Engineering Informatics 24(3) (2010) 367-376.

[2] S. Yang, E. Wei, et al., Triangle chain codes for image matching, Journal of Visual Neurocomputing 120 (10) (2013) $268-276$.

[3] J. Liang, Z. Liao, S. Yang, Y. Wang, Image matching based on orientation-magnitude histograms and global consistency, Pattern Recognition 
45(10) (2012) 3825-3833.

[4] M. Brown, D. Lowe, Automatic panoramic image stitching using invariant features, International Journal of Computer Vision 74 (1) (2007) 59-73.

[5] J. G. Han, D. Farin, P. de With, Broadcast court-net sports video analysis using fast 3-D Camera modeling, IEEE Transactions on Circuits and Systems for Video Technology 18 (11) (2008) 1628-1638.

[6] J. G. Han, D. Farin, P. de With, A mixed-reality system for broadcasting sports video to mobile devices, IEEE MultiMedia 18 (2) (2011) $72-84$.

[7] B. Kratochvil, L. Dong, L. Zhang, B. Nelson, Image-based 3D reconstruction using helical nanobelts for localized rotations, Journal of Microscopy 237 (2) (2010) 122-135.

[8] J. Ishii, S. Sakai, et al., Wide-baseline stereo matching using ASIFT and POC, in: Proceedings of the 19th IEEE International Conference on Image Processing, 2012, pp. 2977-2980.

[9] L. Cheng, J. Gong, M. Li, Y. Liu, 3D building model reconstruction from multi-view aerial imagery and lidar data, Photogrammetric Engineering \& Remote Sensing 77 (2) (2011) 125-139.

[10] Q. Zhang, Y. Wang, L. Wang, Registration of images with affine geometric distortion based on maximally stable extremal regions and phase congruency, Image and Vision Computing 36 (2015) 23-39.

[11] A. Zamir, M. Shah, Image geo-localization based on multiple nearest neighbor feature matching using generalized graphs, IEEE Transaction on Pattern Analysis \& Machine Intelligence 36 (8) (2014) 1546-1558.

[12] S. Wang, H. You, K. Fu, BFSIFT: A novel method to find feature matches for SAR image registration, IEEE Geoscience and Remote Sensing Letters 9 (4) (2012) 649-653.

[13] Y. Zhu, S. Cheng, Image registration using BP-SIFT, Journal of Visual Communication \& Image Representation 24 (4) (2013) $448-457$.

[14] K. Mikolajczyk, C. Schmid, A performance evaluation of local descriptors, IEEE Transaction on Pattern Analysis \& Machine Intelligence 27 (10) (2005) 1615-1630.

[15] G.B. Yao, K.Z. Deng, L.G. Bai, Remote sensing image registration based on Hessian-Affine, Science of Surveying and Mapping 38(3) (2013) 154-156.

[16] J. G. Han, E. J. Pauwels, P. de Zeeuw, Visible and infrared image registration in man-made environments employing hybrid visual features, Pattern Recognition Letters 34 (1) (2013) 42-51.

[17] R. Hartley, A. Zisserman, Multiple View Geometry in Computer Vision, Cambridge University Press, New York, NY, USA, 2006.

[18] T. Suk, J. Flusser, Point-based projective invariants, Pattern Recognition 33 (2000) 251-261.

[19] D. Lowe, Distinctive image features from scale-invariant keypoints, International Journal of Computer Vision 60 (2) (2004) 91-110.

[20] J. M. Morel, G. Yu, ASIFT: A new framework for fully affine invariant image comparison, SIAM Journal on Imaging Sciences 2 (2) (2009) 438-469.

[21] J. Matas, O. Chum, M. Urban, T. Pajdla, Robust wide-baseline stereo from maximally stable extremal regions, Image and Vision Computing 22 (10) (2004) 761-767.

[22] M. Brown, D. Lowe, Invariant features from interest point groups, in: Proceedings of the 13th British Machine Vision Conference, 2002, pp. 656-665.

[23] B. Fan, F. Wu, Z. Hu, Robust line matching through line-point invariants, Pattern Recognition 45 (2012) 794-805.

[24] K. Wang, T. Shi, et al., Image registration using a point-line duality based line matching method, Journal of Visual Communication \& Image Representation 24 (2013) 615-626.

[25] P. Srestasathiern, A. Yilmaz, Planar shape representation and matching under projective transformation, Computer Vision and Image Understanding 115 (2011) 1525-1535.

[26] R. I. Hartley, Theory and Practice of Projective Rectification, International Journal of Computer Vision 35 (2) (1999) 115-127.

[27] C. Papachristou, A. N. Delopoulos, A method for the evaluation of projective geometric consistency in weakly calibrated stereo with application to point matching, Computer Vision and Image Understanding 119 (2014) 81-101.

[28] J. Mallon, P. F. Whelan, Projective rectification from the fundamental matrix, Image and Vision Computing 23 (2005) 643-650.

[29] J. A. Denton, J. R. Beveridge, An algorithm for projective point matching in the presence of spurious points, Pattern Recognition 40 (2007) 586-595.

[30] M. A. T. Figueiredo, R. D. Nowak, S. J. Wright, Gradient projection for sparse representation reconstruction: application to compressed sensing and other inverse problems, IEEE Journal of Selected Topic in Signal Processing 1 (4) (2007) 586-597.

[31] X. Peng, H. Tang, et al., A unified framework for representation-based subspace clustering of out-of-sample and large-scale data, IEEE Transactions on Neural Networks and Learning Systems (2016) 1-14. (in press)

[32] X. Peng, Z. Yi, H. Tang, Robust subspace clustering via thresholding ridge regression, in: 29th AAAI Conference on Artificial Intelligence, 2015, pp.3827-3833.

[33] Z. Zhang, A. Ganesh, X. Liang, Y. Ma, TILT: Transform invariant low-rank textures, International Journal of Computer Vision 99 (1) (2010) 314-328. 
[34] X. Zhang, Z. Lin, F.Sun, et al., Transform invariant text extraction, Image and Visual Computer 30 (4) (2014) $401-415$.

[35] A. Zhang, Y. Matsushita, Y. Ma, Camera calibration with lens distortion from low-rank textures, in: Proceedings of IEEE Conference on Computer Vision and Pattern Recognition, 2014, pp. 2321-2328.

[36] X. Zhang Z. Lin, F. Sun, et al., Rectification of optical characters as transform invariant textures, in: Proceedings of the 12th IEEE International Conference on Document Analysis and Recognition, 2013, pp.393-397.

[37] Y. Peng, A. Ganesh, et al., RASL: Robust alignment by sparse and low-rank decomposition for linearly correlated images, IEEE Transaction on Pattern Analysis \& Machine Intelligence 34(11) (2012) 2233-2246.

[38] J. Canny, A computational approach to edge detection, IEEE Transaction on Pattern Analysis and Machine Intelligence 6 (8) (1986) 679-698.

[39] R.C. Gonzales, R.E. Woods, Digital Image Processing (Third Edition), Pearson Education Asia Ltd., New Jersey, USA, 2011.

[40] S. Belongie, J. Malik, J. Puzicha, Shape matching and object recognition using shape contexts, IEEE Transaction on Pattern Analysis \& Machine Intelligence 24 (24) (2002) 509-522.

[41] Y. Cao, Hong Zhang, et al., Matching image with multiple local features, in: 2010 International Conference on Pattern Recognition, 2010, pp. 519-522. 